\title{
Justificación y consenso global
}

\author{
FEDERICo Matías PAILOS \\ Departamento de Filosofía \\ Universidad de Buenos Aires \\ fpailos@hotmail.com
}

\begin{abstract}
Resumen: Rorty niega que una comunidad pueda equivocarse en la justificación de un enunciado. Afirma que si existe consenso global, no es aceptable una descripción de los estándares de justificación que nos diga que toda la comunidad se equivoca en la justificación de un enunciado, pues no ha considerado todas las prácticas relevantes. Podemos aceptar como criterio de membresía a una comunidad el participar de ciertas prácticas; pero no existe un criterio que nos permita identificar las prácticas relevantes para poder fijar la extensión de la comunidad. Esto nos permite imaginar una situación en la cual el consenso global no es condición suficiente para determinar que un enunciado está justificado para esa comunidad. Pero de un ejemplo similar podemos desestimar la posibilidad de negarle sentido a la idea de que toda una comunidad puede equivocarse con respecto a la justificación de enunciados.
\end{abstract}

Palabras clave: comunidad, enunciado, acuerdo global, práctica

En el debate que en el curso de estos últimos años han mantenido Rorty y Putnam en torno al problema de la justificación de enunciados y creencias, Putnam ha afirmado que una de las características de la justificación es la de ser independiente de la opinión de la mayoría de los miembros de una comunidad. Esto es, una mayoría puede estar equivocada. Ciertamente, si existe un miembro de la comunidad cuyo dictamen acerca de la justificación de un enunciado difiere de aquel del resto, continuaría abierto el juego de dar y pedir razones; y esto lo reconoce Rorty. Pero, ¿puede toda la comunidad estar equivocada? ${ }^{1}$ Aquí la respuesta es más problemática.

Prima facie, y asumiendo que los estándares de justificación de esa comunidad surgen de sus prácticas (están implícitos en en ellas), podríamos reconocer sus estándares de justificación y comprobar que, siguiéndolos rigurosamente, el enunciado que ellos (los miembros de la comunidad) creían justificado, esto es, que creían que satisfacía sus propios estándares de justificación, en realidad no los satisfacía. En consecuencia, el consenso global no es índice suficiente de la justificación. Ésta es la posición que defiende Robledo. ${ }^{2}$

${ }^{1}$ Tal como señala Penelas ("La justificación como hecho social", pp. 127-134, en este mismo número), Rorty no cree que el consenso mayoritario sea condición suficiente para atribuir justificación. Pero sí creen, tanto Rorty como Penelas, que el consenso global lo es.

${ }^{2}$ L. Robledo, “iPuede realmente una mayoría estar equivocada?”, pp. 143-149, en este mismo volumen. 
Esta interpretación recoge la idea que señala la diferencia entre creer que se sigue una norma y seguirla de hecho. ${ }^{3}$ Aunque los individuos afirmen que creen $X$, si su conducta se explica mejor atribuyéndoles la creencia en no $X$, debemos (es más razonable) atribuirles la creencia en no $X$. Aplicado a nuestro ejemplo, aunque el conjunto de los miembros de la comunidad afirmen que $X$ está justificada (porque cumple con sus estándares de justificación), en verdad $X$ no cumple con todos esos estándares de justificación, y en consecuencia no está justificado.

Pero podría darse otra lectura de esta situación. Sus afirmaciones forman parte de sus prácticas. En consecuencia, una atenta interpretación de esas prácticas tendría que dar cuenta de esa conducta. Es decir, los estándares de justificación que les atribuimos (y que suponemos implícitos en sus prácticas) deben construirse a partir de toda la conducta relevante. Es de suponer que sus dichos acerca de si un enunciado está o no justificado son parte de la conducta relevante que ha de considerarse. Con lo cual, podemos afirmar, los estándares de justificación que la primera interpretación le atribuye a esa comunidad no eran los que la comunidad en verdad sostiene. Y de acuerdo con los verdaderos estándares de justificación (que toman en cuenta la práctica efectiva de los miembros de la comunidad —que incluye el intercambio lingüístico-), el enunciado en cuestión estaba justificado.

Como vemos, estas diferentes reconstrucciones de la situación determinan cuáles estándares de justificación le atribuimos a la comunidad considerada; pero las reconstrucciones, a su vez, dependen de qué prácticas recojamos (decidamos considerar como relevantes) para atribuir estándares de justificación a esa comunidad.

Pensemos en el ejemplo expuesto por Barrio. ${ }^{4}$ Supongamos una comunidad de matemáticos que creen haber resuelto un problema matemático sin solución hasta ese momento. Una consideración atenta a las prácticas (que nos parezcan relevantes para el caso) que reconocemos en esa comunidad (incluidas sus afirmaciónes acerca de sus estándares de justificación) arrojó como resultado que no creamos que la solución propuesta por esa comunidad satisfaga los estándares de justificación propios de ella. Es decir, de acuerdo con sus propios estándares de justificación, ese problema no estaba resuelto (la prueba que ellos creían haber ofrecido violaba uno de los estándares de justificación relevantes). Supongamos que no es posible presentarles la prueba de su error (por ejemplo, por ser una comunidad del pasado). ¿Cómo interpretar esta nueva situación?

\footnotetext{
${ }^{3}$ Wittgenstein introduce esta distinción en el § 202 de las Investigaciones filosóficas (Philosophical Investigations, Basil Blackwell, Oxford, 1953 [versión en castellano: Investigaciones filosóficas, Instituto de Investigaciones Filosóficas-UNAM/Crítica, Barcelona, 1988]).

${ }^{4}$ E. Barrio, "Todos ellos no son los que pueden justificarnos", pp. 167-173, en este número.
} 
1) Aceptemos como criterio de membresía a una comunidad, el participar en ciertas prácticas. ${ }^{5}$ Puede decirse que yo comparto con la comunidad en cuestión ciertos estándares de justificación del tipo relevante para el caso y en número suficiente como para considerárseme miembro de la comunidad (por ejemplo, el siguiente estándar de justificación: si un problema matemático de tipo $A$ está solucionado si y sólo si cumple las condiciones $B$ y $C$, y no se cumple alguna de ellas, entonces el problema no está solucionado). En este caso, yo formo parte de la comunidad y, por tanto, no existe consenso global. Con lo que este ejemplo no sirve para evaluar si un enunciado puede no estar justificado aunque exista un consenso global en que lo está. Una interpretación como ésta, aunque más refinada (que incluye, por ejemplo, la distinción entre consenso global y consenso global racional), es la que defiende Barrio. ${ }^{6}$

2) Pero, aun aceptando aquel criterio de membresía a una comunidad (el participar de ciertas prácticas), podemos decir que existen prácticas que todos ellos comparten, y yo no. Y de estas prácticas se deriva el siguiente estándar de justificación: la solución de un problema matemático está justificado si y sólo si cumple con las condiciones $B$ y $C$. Ellos (y no yo) conforman una particular comunidad matemática, porque comparten esta práctica (que yo, por hipótesis, no comparto). Dentro de esa comunidad existe consenso global en que el problema está solucionado (esto es, que satisface las condiciones $B$ y $C$ ). Nosotros vemos que eso no es así. En consecuencia, existe un consenso global de que el problema está solucionado, cuando en verdad, de acuerdo con sus propios estándares de justificación, no lo está. El consenso global no es, entonces, índice de justificación (como señalamos, ésta es la interpretación que prefiere Robledo).

Rorty y Putnam concuerdan en que podemos ver que una comunidad se define a partir de las prácticas que consideremos relevantes (que dependen a su vez, por lo menos, de los intereses que guíen nuestro análisis). Diferentes prácticas determinan distintas comunidades (un mismo conjunto de individuos puede participar de diferentes comunidades). El problema, entonces, pasa por encontrar un criterio que permita distinguir las prácticas relevantes, a partir de las cuales distinguir una comunidad, y de allí,

${ }^{5}$ Seguimos, en este punto, la idea de Cresto ("Justificar, interpretar y otros asuntos pragmáticos", pp. 151-158, en este número.), quien define así la pertenencia a una comunidad. Qué prácticas se considerarán relevantes varía de caso en caso, de acuerdo con el tipo de comunidad considerada. La pertenencia a la comunidad de los matemáticos queda definida por un tipo diferente de prácticas a las que define a pertenencia a la comunidad de los futbolistas.

${ }^{6}$ E. Barrio, op. cit. 
extractar sus estándares de justificación. ¿Existe un criterio de estas características?

Un criterio obvio parece ser el que toma en consideración el conjunto de la conducta de los individuos considerados. Pero ya vimos que estas conductas pueden recibir diferentes interpretaciones - una misma conducta puede ser considerada relevante por una interpretación e irrelevante por otra, o puede variar el grado de relevancia que le otorguen distintas interpretaciones. Así, en el ejemplo anterior podemos tomar ciertas conductas linguísticas como índice de asentimiento, y de esta forma concluir que existe consenso global y que, por tanto, el consenso global no es condición suficiente que indique la existencia de justificación. Pero podemos reinterpretar esa conducta lingüística de modo tal que no sea un índice de asentimiento (por ejemplo, podemos imaginar una sociedad que permita la contradicción en el discurso - porque nada de lo que se diga se cree si no está acompañado de cierta conducta no lingüística- y que toma como índices de asentimiento otras conductas, por ejemplo, hacer una reverencia). Sólo podemos hablar de la existencia de un consenso global una vez que hayamos fijado la traducción de los índices de asentimiento; pero ésta nunca deja de ser una hipótesis interpretativa. Supuesta esta hipótesis, en efecto, podemos hablar de la existencia de un consenso global sin que exista justificación. Como afirma Cresto, ${ }^{7}$ no sólo debemos señalar que un enunciado está justificado para una comunidad $A$; debemos también hacer notar que ese enunciado está justificado para esa comunidad $A$ según una comunidad $Z$, donde $Z$ puede o no ser la propia $A$. En el caso de que $Z$ no sea igual a $A$, podemos decir que un enunciado $x$ no está justificado para $A$ según $Z$ aunque exista en $A$ consenso en que $x$ está justificado.

Quisiera hacer un último comentario relacionado con la posibilidad de señalar la independencia de la justificación del consenso global y con la posibilidad de conciliar esta posición con aquella que afirma que los estándares de justificación surgen de las prácticas. Analizando las prácticas de la primera comunidad, podemos llegar a explicitar estándares de justificación vigentes en ella. ${ }^{8}$ Uno de ellos puede ser explicitado por medio del siguiente enunciado: un enunciado $D$ está justificado si y sólo si cumple con las condiciones $A$ y $B$. Si $D$ cumple $A$ y $B$, los miembros de la comunidad van a estar justificados al asentir a $D$. Podemos imaginar una situación, en el tiempo 1 , en la que, luego de un periodo de argumentación y contraargumentación (de cumplir con el juego de dar y pedir razones), el conjunto de los miembros de la comunidad asienten al enunciado $D$. Luego, en el tiempo 2 , uno de ellos afirma que no se cumplió con uno de los requisitos

${ }^{7}$ E. Cresto, "Justificar, interpretar y otros asuntos pragmáticos", p. 155, en este número.

${ }^{8}$ La idea de "explicitación" de las normas de una comunidad es presentada por R. Brandom en Making It Explicit, Harvard University Press, Cambridge, 1994. 
de los estándares de justificación pertinentes. Todos concuerdan con él. ¿Cómo describir esta nueva situación?

Nuevamente podemos hacerlo de diferentes maneras. Podemos afirmar, por ejemplo, que nunca existió consenso global, porque a la comunidad considerada pertenecen tanto el conjunto de individuos del tiempo 1, como el conjunto de los individuos del tiempo 2. Y los individuos del tiempo 2 no creen lo que creían los individuos del tiempo 1. En consecuencia, no existe consenso global. Ésta es una posible descripción de la situación.

Otra posibilidad es sostener que, a pesar de que existe consenso global (todos los individuos del tiempo 1 estaban de acuerdo), el enunciado no satisfacía los estándares de justificación de esa comunidad (como ahora, en el tiempo 2, vemos claramente).

Como conclusión: si no existe un criterio independiente que nos obligue a optar por una u otra descripción de la situación (es decir, quiénes forman parte de la comunidad), no se puede concluir que la justificación es independiente del consenso global, ni que no lo es. La evaluación que hagamos depende de quiénes son los miembros de la comunidad, de cuáles son la prácticas relevantes a partir de las cuales extraer los estándares de justificación. Pero podemos, entonces, pensar una descripción en la cual el consenso global es independiente de la justificación (donde existe consenso global, pero no justificación). Si esto es suficiente para afirmar la independencia lógica entre consenso global y justificación, toda una comunidad puede estar equivocada con respecto a la justificación de un enunciado.

Recibido el 19 de junio de 2002; aceptado el 26 de noviembre de 2002 